\title{
Automated image processing for quantification of blue-stain discolouration of Norway spruce wood
}

\author{
M. Schubert • S. Mourad • F. W. M. R. Schwarze
}

Received: 3 April 2009/Published online: 11 April 2010

(C) Springer-Verlag 2010

\begin{abstract}
Bioincising is a promising method for enhancing liquid uptake (e.g. preservatives or wood-modification agents) in refractory wood. Incubation with the white-rot fungus, Physisporinus vitreus, which selectively degrades pit membranes, results in deeper and more homogeneous penetration of liquids. Conventional methods of assessing the degree of fungal discolouration of wood after treatment with preservatives (e.g. European standard EN 152) are partly based on a subjective rating scale, which gives a rough value of the surface colonisation by blue-stain fungi. Hence, an automated image processing (AIP) procedure was developed for standardised quantification of the segmentation thresholds of discolouration and tested against manual segmentation analysis. Using the red filter in the AIP method revealed high correlation $\left(R^{2} 0.95\right)$ and allowed for more user friendly and objective determination of blue staining of wood.
\end{abstract}

\section{Introduction}

This study is part of a larger study focusing on the effect of wood preservatives against blue staining of bioincised and non-treated heartwood of Norway spruce. The bioincising process using Physisporinus vitreus is a promising approach to improve the uptake of preservatives and wood-modification substances by refractory wood. Based on the findings from previous laboratory investigations (Schwarze

M. Schubert $(\bowtie) \cdot$ F. W. M. R. Schwarze

Group of Wood Protection and Biotechnology, Wood Laboratory,

Swiss Federal Laboratories for Materials Testing and Research (EMPA),

Lerchenfeldstrasse 5, 9014 St Gallen, Switzerland

e-mail: mark.schubert@empa.ch

S. Mourad

Program Manager Technologies, Engineering, Fisba Optik AG,

Rorschacherstrasse 268, 9016 St Gallen, Switzerland 
et al. 2006), an industry cooperation project was launched with the objective of scaling up the bioincising process. One requirement of the project was to analyse a large number of wood blocks treated by several preservatives against blue staining. The European Standard EN 152 (1984) is widely accepted for testing blue-stain resistance of treated wood.

EN 152 describes a method of estimating the degree of blue staining on the surface of wood using a rating scale from 0 (none) to 3 (severe), which, because of the subjectivity inherent in this type of valuation, is one shortcoming of the technique. Therefore, more accurate and faster methods of assessment need to be evaluated to make intra- and interlaboratory results more comparative. The aim of the present study was to develop a simple method of automated image processing (AIP) that would enable an objective numeric assessment of blue-stain discolouration, thus reducing subjectivity.

\section{Materials and methods}

Preparation of the material

Bioincised $(n=70)$ and non-treated $(n=70)$ heartwood samples $(30 \times 40 \times$ $15 \mathrm{~mm}^{3}$, length $\times$ width $\times$ height) of Norway spruce (Picea abies) were treated with seven wood preservatives, all of them commercial products. Norway spruce wood without preservatives was used for assessing the virulence of the tested fungi in bioincised $(n=40)$ and non-treated $(n=40)$ blocks. Liquids were applied to the wood blocks by either dipping (30 min) or vacuum impregnation (20 min, 7 mbar). The wood blocks were conditioned for 2 weeks at $20^{\circ} \mathrm{C}$ and $65 \%$ relative humidity and then exposed to a mixed fungal suspension of Discula pinicola (DSM-4948), Ceratocystis picea (DSM-4842), Cladosporium herbarum (DSM-63422), Aureobasidium pullulans (EMPA-316), and Sclerophoma pityophila (EMPA-315) for 6 weeks.

\section{Image processing}

Images were acquired with an image acquisition system (eVision executive, Kaiser, Germany) and digital colour camera (Nikon Coolpix 990). The wood samples were illuminated with two parallel lamps (length $60 \mathrm{~cm}$ ) for uniform light intensity and the camera was located vertically at a distance of $25 \mathrm{~cm}$ from the sample. Images (TIFF format) were created with the maximum resolution $(2,048 \times 1,536$ pixels $)$.

Several classifying algorithms were tested and evaluated with respect to their use as quantifiers for blue-stain discolouration (Fig. 1).

Manual segmentation (MS) was performed using the roipoly function (specify polygonal region-of-interest) in Matlab ${ }^{\circledR}$ (Version 7.4 R2007a, MathWorks, Natick, MA, USA) in order to evaluate the results of the manual threshold (MT) and AIP methods described below. This function was used to delineate the areas of fungal discolouration using the mouse, thus creating a manual segmentation of the sample.

The MT method involves separate interactions for every image and requires a threshold input. The image is displayed on the screen, converted to an 8-bit 


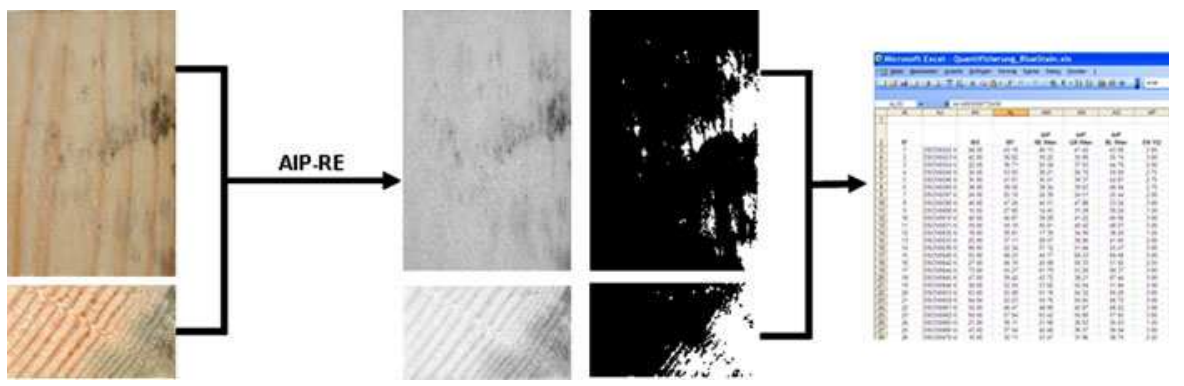

Fig. 1 Scheme of automated image processing using red filter (AIP-RE). Conversion of a RGB image to a high-contrast grey-scale and a binary image for quantification of the area of discolouration

grey-scale image and a threshold value for separating fungus from wood is selected. Most image analysis softwares (e.g. ImageJ) support this feature.

The AIP method was developed using Matlab code. The algorithm for segmentation includes: (1) testing of conversion of the red-green-blue (RGB) image to a high-contrast grey-scale image using the red (RE), green (GR) and blue (BL) filters separately; (2) computation of a global threshold using Otsu's method (minimising the intra-class variance of the threshold black and white pixels) (Otsu 1979); and (3) morphological operations in order to fill the possible artificial holes and to remove small objects (artefacts) present in the segmented binary image.

The correlation coefficients between MS and AIP-RE, GR, BL or MT were calculated in order to evaluate the performance of the image processing methods. Different results for each specimen (i.e. difference in discolouration percentage: DDP) between MS and MT or AIP-RE, GR, BL were calculated according to Künninger (2008): $\Delta$ DDP $=$ MS-MT/AIP-RE, GR, BL (\%).

\section{Results and discussion}

Because most of the current methods of assessing fungal discolouration of wood are based on subjective evaluation of the wood surface (e.g. EN 152), there is much interest in developing a method that enables a more objective, and thus comparative, expression of the visual evaluation of blue-stain discolouration (Ważny and Rudniewski 1972; Viitanen and Ritschkoff 1991; Clausen and Yang 2005; Kartal et al. 2005). There are some successful approaches for the detection and quantification of wood surface defects or wood texture applying fractal dimension of the triangular prism surface approach or by methods based on artificial neural networks (Liu and Furuno 2001, 2002; Ruz et al. 2005). Van den Bulcke et al. (2005) was the first to investigate artificial neural networks and the triangular prism method to detect and quantify blue-stain discolouration on wood. Both methods showed good results and a high correlation but are complex and difficult for nonprofessionals to apply. Thus, the aim of the present study was to develop a userfriendly, rapid image processing procedure that does not require prior configuration 
and enables objective determination of the percentage of blue-stain discolouration on bioincised and non-treated Norway spruce wood. The segmentation procedure of the discoloured wood using the MT and AIP-RE, GR, BL methods is displayed in Figs. 2, and 3. Threshold techniques are applicable for grey-scale (two-dimensional) images and usually a high-contrast grey-scale image is converted from an optimal linear combination of the RGB colour components (Xavier et al. 2001; Mery and Pedreschi 2005). In this study, separate filter images were used to select the optimum filter and threshold technique, and it was found that the RE filter resulted in a high-contrast grey-scale image (Fig. 2). The RE filter minimised the density (colour) difference between early wood and the (reddish) latewood by filtering the red tones, creating a binary image in which quantification of the area of blue staining was readily achieved without the negative influence (i.e. artefacts) of the dark (reddish) latewood cells (Fig. 3).

Plotting the results of MS and the AIP demonstrated a particularly high correlation for AIP-RE (Fig. 4a). The coefficient of determination, $R^{2}$, for AIP-RE was 95.85, and the descending order of the coefficients of each method was AIP-RE (95.85) > MT (87.42) > AIP-GR (86.05) and AIP-BL (77.42).

Calculation of the mean DPP for each image processing method resulted in the following values: MS-MT $=-8.36 \pm 11.06$; MS-AIP-RE $=+0.19 \pm 5.77$; MSAIP-GR $=-8.45 \pm 11.33$; MS-AIP-BL $=-12.44 \pm 13.61$. This result implied that all methods, with the exception of AIP-RE, exaggerated the degree of blue-stain discolouration because of misinterpretation of dark latewood, expressed as negative DPP values.

The results of the performance of the preservatives used on bioincised and nontreated wood will be reported separately. However, there was no significant effect of the preservatives or of bioincised wood on the performance of the image processing procedures used. Generally, the visual results and the statistical analysis showed that the AIP-RE procedure is an appropriate method of quantifying blue-stain discolouration of Norway spruce wood. In this context, it must be stressed that the results are only valid for the sap- and heartwood of Norway spruce, and that
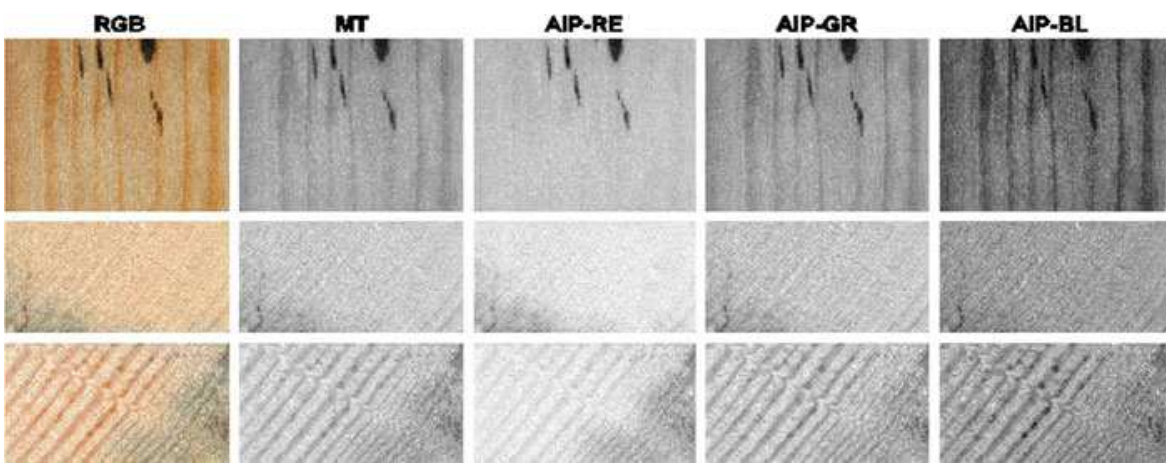

Fig. 2 Visual comparison of converted grey-scale images by the image analysis method's manual threshold (MT) and automated image processing using red (RE), green (GR) and blue (BL) filters (AIP(RE, GR, BL)) 


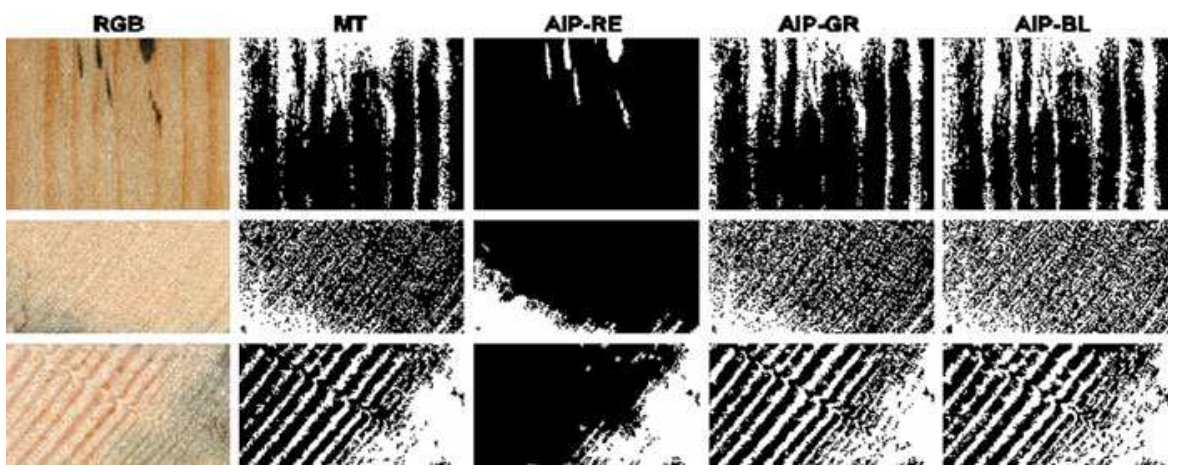

Fig. 3 Visual comparison of binary images generated by manual threshold (MT) image processing and the automated image processing procedure using red (RE), green $(\mathrm{GR})$ and blue (BL) filters (AIP-(RE, GR, BL))

(a)

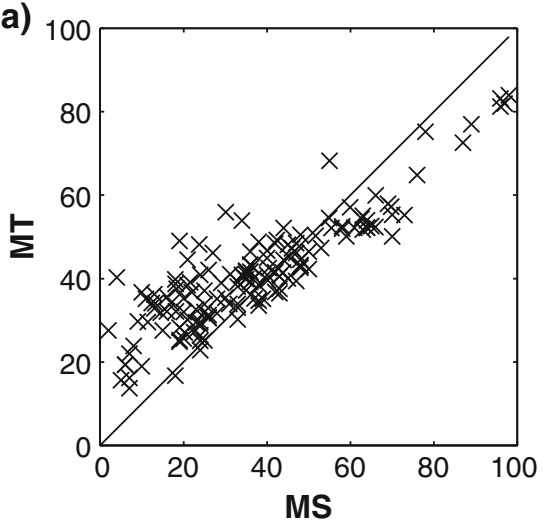

(c)

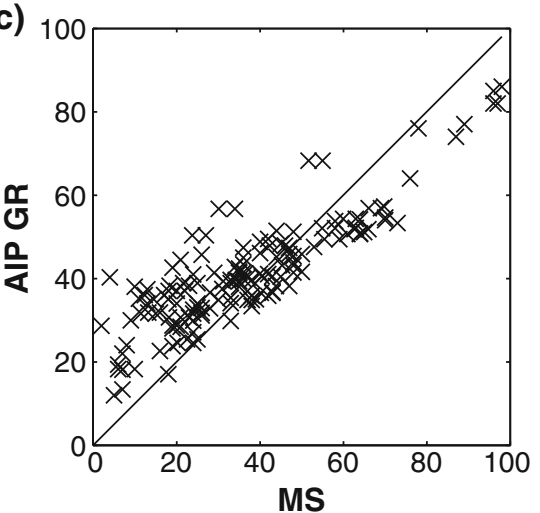

(b)

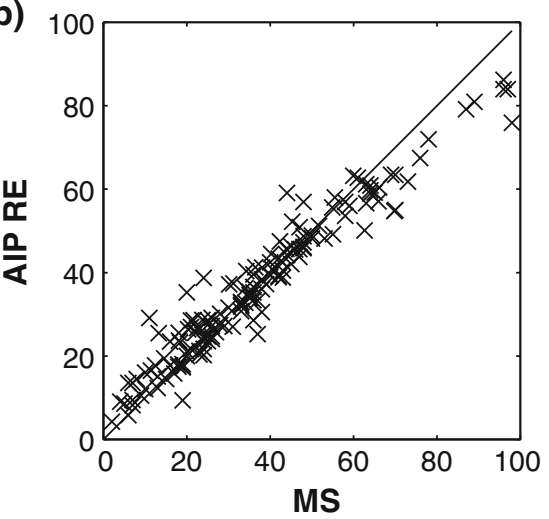

(d)

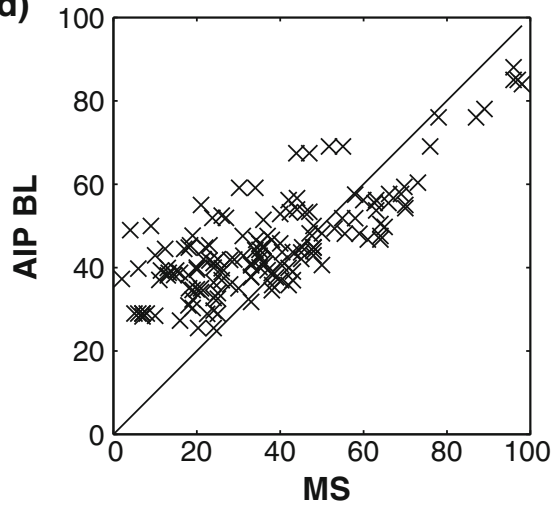

Fig. 4 Comparison of results of numeric assessment of blue-stain discolouration by manual segmentation (MS) and manual threshold (MT) (a), AIP-RE (b), AIP-GR (c) and AIP-BL (d). AIP $=$ automated image processing 
studies on other wood species are still in progress. However, there are reasons to believe that AIP-RE will provide similar good results for other lighter softwoods (e.g. pine and fir).

\section{Conclusion}

A computer-based AIP procedure for quantifying blue-stain discolouration of bioincised and non-treated wood of Norway spruce is described. The automated image method includes conversion of the RGB image to a high-contrast grey-scale image using the RE, GR and BL filters separately. Computation of a global threshold is based on Otsu's method and morphological operations are performed in order to optimise the binary image. Visual and statistical results show that segmentation accuracy is better when using the red filter (AIP-RE). AIP-RE revealed a high correlation $\left(R^{2} 95.85\right)$ and a DPP value near zero (0.19).

The AIP procedure described here can be used for determining blue-stain discolouration of Norway spruce wood for quality control in the wood protection industry and/or for research purposes. Further studies are currently in progress to validate and enhance the potential of the AIP-RE method for other wood species.

Acknowledgments The present work emerged from the research project CTI-8593.1 LSPP-LS 'Bioincising of conifer wood with Physisporinus vitreus to improve its treatability for a range of wood preservation and modification methods'. The authors express their gratitude to the Swiss CTI (Innovation Promotion Agency) for its financial support.

\section{References}

Clausen CA, Yang VW (2005) Azole-based antimycotic agents inhibit mold growth on unseasoned pine. Int Biodeter Biodegrad 55:99-102

European Committee for Standardization (1984) Standard EN 152: test methods for wood preservatives. Laboratory method for determining the protective effectiveness of preservative treatment against blue stain in service

Kartal SN, Brischke C, Rapp AO, Imamura Y (2005) Biological effectiveness of didecyl dimethyl ammonium tetrafluoroborate (DBF) against basidiomycetes following preconditioning in soil bed tests. Wood Sci Technol 40:63-71

Künninger T (2008) A semi-automatic method to determine the wood failure percentage on shear test specimens. Holz Roh Werkst 66:229-232

Liu J, Furuno T (2001) The fractal evaluation of wood texture by the triangular prism surface area method. Wood Fiber Sci 33:213-222

Liu J, Furuno T (2002) The fractal estimation of wood colour variation by the triangular prism surface area method. Wood Sci Technol 36:385-397

Mery D, Pedreschi F (2005) Segmentation of colour food images using a robust algorithm. J Food Eng $66: 353-360$

Otsu N (1979) A threshold selection method from gray-level histograms. IEEE Trans Syst Man Cybern 9:62-66

Ruz GA, Estévez PA, Perez CA (2005) A neurofuzzy colour image segmentation method for wood surface defect detection. Forest Prod J 55:52-58

Schwarze FWMR, Landmesser H, Zgraggen B, Heeb M (2006) Permeability changes in heartwood of Picea abies and Abies alba induced by incubation with Physisporinus vitreus. Holzforschung 60:450-454 
Van den Bulcke J, Acker JV, Stevens M (2005) Image processing as a tool for assessment and analysis of blue stain discolouration of coated wood. Int Biodeter Biodegrad 56:178-187

Viitanen H, Ritschkoff A (1991) Mould growth in pine and spruce sapwood in relation to air humidity and temperature. Department of Forest Products, The Swedish University of Agricultural Scienses, Uppsala, Report no 221

Ważny J, Rudniewski P (1972) The biodeterioration of binding materials used in artistic painting. Mater Org 7:81-92

Xavier JB, Schnell A, Wuertz S, Palmer R, White DC, Almeida JS (2001) Objective threshold selection procedure (OTS) for segmentation of scanning laser confocal microscope images. J Microbiol Methods 47:169-180 